and sarcoma), who underwent PE with curative intention at our institution between 1999 and 2021.

Overall survival (OS) was assessed with Kaplan-Meier analysis. Differences in survival according to type of PE (anterior, posterior or total) were assessed using log-rank tests. Multivariate analysis using Cox-proportional hazard models was performed to determine the impact of age on survival outcomes. Results were considered statistically significant when $\mathrm{p}<0,05$.

Result(s)* In total, 56 patients were included in the study. Average age at the time of surgery was 58,3 (SD 14,4) years. Distribution of tumor site was: ovary $(n=32)$, cervix $(n=10)$, endometrium $(n=8)$, vulva/vagina $(n=6)$. PE was anterior $(n=6)$, posterior $(n=31)$ or total $(n=19)$.

One case of intraoperative complication was observed, with a vascular lesion that was sutured with no further aggravation. Overall, morbidity occurred in 46,4\% of patients; $18(32,1 \%)$ developed early complications and $8(14,3 \%)$ developed at least one late complication, including 7 gastrointestinal, 3 urinary, 4 incision hernias and 11 infectious complications. Fourteen cases required reintervention due to complications. Only one case of early postoperative death was recorded.

The 5-year OS in remaining cases was 31\%. Median follow-up time was 22,5 months (range: 1-242). Mean overall survival tended to be longer for patients with anterior PE (150,6 months, SD 49,5) than for patients with posterior PE (51,0 months, SD 11,3) or total PE (41,2 months, SD 14,3), although this difference was not statistically significant $(p=0,113)$. The 5 -year OS was $60 \%, 25 \%$ and $25 \%$, respectively. Age had no impact on survival.

Conclusion* PE is still the only curative option for selected patients, when chemoradiation and/or primary surgery failed in recurrent or persistent gynecological malignancies. When performed by expert gynecologic-oncology surgeons, this intervention has an acceptable survival and perioperative morbidity rate.

\section{LOCAL ANDROGEN SYNTHESIS AND METABOLISM IN ENDOMETRIAL AND OVARIAN CANCER}

M Gjorgoska*, R Pavlič, T Lanišnik Rižner. Institute of Biochemistry and Molecular Genetics, Faculty of Medicine, University of Ljubljana, Ljulbjana, Slovenia

\subsection{6/ijgc-2021-ESG0.275}

Introduction/Background* Endometrial (EC) and ovarian cancer $(\mathrm{OC})$ are female pathologies that express androgen receptor (AR) to different extents, however, the role of the AR ligands, i.e., androgens and their 11-oxygenated metabolites in EC and OC onset and progression is poorly understood. Active androgens can form from the abundant precursor dehydroepiandrosterone sulfate (DHEA-S) from the adrenal cortex; however, local synthesis and metabolism in endometrial and ovarian cancer tissue may have important roles as well.

Objective To explore the local androgen synthesis and metabolism in EC and OC.

Methodology Gene expression of key enzymes involved in the androgen synthesis and metabolism was examined in model cell lines of type I and II EC, and high grade serous OC. The Cancer Genome Atlas (TCGA) database was searched for the expression of these genes in EC and OC tissues using the UCSC Xena platform.

Result(s)* Our gene expression data indicate that model cell lines of EC and $\mathrm{OC}$ can potentially synthesize the potent androgens testosterone $(\mathrm{T})$ and $5 \alpha$-dihydrotestosterone $(5 \alpha$ DHT) from DHEA-S, as well as the equally potent metabolites 11-keto-T and 11-keto-DHT from the adrenal precursor 11$\mathrm{OH}$-androstendione. Interestingly, the expression of $A K R 1 C 3$, encoding an aldo-keto reductase family enzyme that catalyses the formation of $\mathrm{T}$ and 11-keto-T, and the expression of $S R D 5 A 1$, encoding a steroid $5 \alpha$-reductase that catalyses the conversion of $\mathrm{T}$ to $5 \alpha$-DHT were higher in certain model cell lines of EC and OC comparing to the respective controls. According to the TCGA database, SRD5A1 expression correlates with a worse overall survival in EC, whereas HSD11B2 expression with a positive progression free survival in OC.

Conclusion* Gene expression analysis indicate that active androgens and 11-oxyandrogens can form in EC and OC. Further metabolism studies are in progress. Assessment of the effect of androgens and their metabolites on EC and OC development and determination of their systemic levels may unravel novel therapeutic targets and new diagnostic and prognostic biomarkers.

This work was supported by the Slovenian Research Agency. Grant number: J3-2535.

\section{PROGNOSTIC FACTORS AND SURVIVAL OF PATIENTS WITH UTERINE SARCOMA - A GERMAN UNICENTRIC ANALYSIS}

A Huß*, M Klar, MF Hasanov, I Juhasz-Böss, M Bossart. Faculty of Medicine University Freiburg, Obstetrics and Gynaecology, Freiburg im Breisgau, Germany

\subsection{6/ijgc-2021-ESGO.276}

Introduction/Background* Uterine sarcoma (US) as a histologically heterogeneous group of tumours is rare and associated with a poor prognosis. This study aimed to describe the survival and identify prognostic variables in patients with US.

Methodology This unicentric, retrospective cohort study includes 57 patients with US over 18 years treated at the Department of Obstetrics and Gynaecology at University Hospital Freiburg, Germany between 1999 and 2017. Progressionfree survival (PFS) and overall survival (OS) were calculated and visualised in Kaplan-Meier curves. Prognostic factors for total cohort and Leiomyosarcoma (LMS) patients were identified using log-rank test and Cox-regression.

Result(s)* 44 LMS, seven low grade-endometrial stromal sarcoma (LG-ESS), four high grade-ESS and two undifferentiated US patients were identified. The median age at time of diagnosis was 51.0 years (range 18-83). The median follow-up time was 35 months. PFS for the total cohort was 14.0 months (95\%-Confidence-Interval (CI) 9.7-18.3) and OS 36.0 months (95\%-CI 22.1-49.9). Tumour pathology was prognostically significant for OS with LG-ESS being the most favourable (mean OS 150.3 months). In the multivariate analysis, patients over 52 years showed a four times higher risk for tumour recurrence (hazard ratio (HR) 4.4; 95\%-CI 1.5-12.9). Progesterone receptor negativity was associated with a two times higher risk for death (HR 2.8; 95\%-CI 1.0-7.5). For LMS patients in the univariate analysis young age $(p=0.04)$, clear surgical margins $(p=0.008)$, low FIGO stage $(p=0.01)$ and no application of chemotherapy $(p=0.02)$ were statistically significant positive factors for OS.

Conclusion* In this analysis, tumour histology, age at time of diagnosis and progesterone receptor status were prognostic factors for US. Unfavourable OS in LMS patients was 